\title{
Probabilistic set-membership state estimator
}

\author{
Luc Jaulin \\ ENSIETA, DTN, 2 rue François Verny, 29806 Brest. \\ Email : jaulinlu@ensieta.fr
}

\begin{abstract}
Interval constraint propagation methods have been shown to be efficient, robust and reliable to solve difficult nonlinear bounded-error state estimation problems. However they are considered as unsuitable in a probabilistic context, where the approximation of a probability density function by a set cannot be accepted as reliable. This paper proposes to new probabilistic approach which makes possible to use classical set-membership observer which are robust with respect to outliers. The approach will be illustrated on a localization of robot in situations where there exist a large number of outliers.
\end{abstract}

\section{Index Terms}

Interval analysis, probability, robust controller, set-membership estimation, outliers.

\section{INTRODUCTION}

Consider the discrete-time dynamic system described by the following nonlinear state equations

$$
\begin{cases}\mathbf{x}(k+1) & =\mathbf{f}_{k}(\mathbf{x}(k), \mathbf{n}(k)) \\ \mathbf{y}(k) & =\mathbf{g}_{k}(\mathbf{x}(k)),\end{cases}
$$

where $\mathbf{x}$ is the state vector, $\mathbf{n}$ is the state noise and $k$ is the time. Since the evolution function $\mathbf{f}$ depends on $k$, this formulation encloses situations where the state equations are time dependent or when the system depends on some known inputs. In a bounded-error context, we generally assume that $\mathbf{n}(k)$ and $\mathbf{y}(k)$ 
belong to some prior feasible sets denoted by $\mathbb{N}(k)$ and $\mathbb{Y}(k)$, respectively. The sets $\mathbb{N}(k)$ are known a priori and the sets $\mathbb{Y}(k)$ are obtained from the measurement vector $\tilde{\mathbf{y}}(k)$ of the output vector $\mathbf{y}(k)$ and take into account some bounded-error noises that could corrupt the measurements. The feasible set $\mathbb{X}(k)$ corresponding to the set of all state vectors $\mathbf{x}(k)$ that are consistent with the past can be computed recursively [2] as follows

$$
\mathbb{X}(k+1)=\mathbf{f}_{k}\left(\mathbb{X}(k) \cap \mathbf{g}_{k}^{-1}(\mathbb{Y}(k)), \quad \mathbb{N}(k)\right)
$$

In this formula, the operations have to be understood in a set-theoretical sense, i.e., $\mathbf{g}_{k}^{-1}(\mathbb{Y})=\left\{\mathbf{x} \mid \mathbf{g}_{k}(\mathbf{x}) \in \mathbb{Y}\right\}$ and $\mathbf{f}_{k}(\mathbb{X}, \mathbb{N})=\left\{\mathbf{z} \mid \exists \mathbf{x} \in \mathbb{X}, \exists \mathbf{n} \in \mathbb{N}, \mathbf{z}=\mathbf{f}_{k}(\mathbb{X}, \mathbb{N})\right\}$

In practice, it may happen that some of the $\mathbf{y}(k)$, the actual value of the output vector at time $k$, do not belong to their corresponding sets $\mathbb{Y}(k)$. The vector $\mathbf{y}(k)$, is said to be an inlier if $\mathbf{y}(k) \in \mathbb{Y}(k)$ and an outlier otherwise. Set-membership methods have been shown to be adapted to deal with problems involving with outliers (see, e.g., [15], [11], [16], [9]). Moreover, when the model is nonlinear, interval constraint propagation methods [14], [18] have been shown to be very efficient and reliable for several state estimation problems (see, e.g., [7], [10], [17], [1] or [5]). The main contribution of this paper is to give a probabilistic interpretation of set-membership observers which makes possible to use set-membership approaches to solve state estimation problems that are expressed in a probabilistic form.

Section II presents a set membership observer which is robust with respect to outliers. Section III provides some probabilistic properties of the observer. An illustrative application is given in Section IV. Section V concludes the paper.

\section{RobUST STATE ESTIMATOR}

In a set-membership context, estimators that are robust with respect to outliers can be obtained by using the notion of relaxed intersection. The $q$-relaxed intersection of $m$ sets $\mathbb{X}_{1}, \ldots, \mathbb{X}_{m}$ of $\mathbb{R}^{n}$ denoted $\{q\}$

by $\bigcap \mathbb{X}_{i}$ is the set of all $\mathbf{x} \in \mathbb{R}^{n}$ which belong to all $\mathbb{X}_{i}$ 's, except $q$ at most. Figure 1 illustrates this notion for $m=6$ and $q=2,3,4$. For this example, we have

$$
\bigcap^{\{0\}} \mathbb{X}_{i}=\bigcap^{\{1\}} \mathbb{X}_{i}=\emptyset, \bigcap^{\{5\}} \mathbb{X}_{i}=\bigcup \mathbb{X}_{i} \text { and } \bigcap^{\{6\}} \mathbb{X}_{i}=\mathbb{R}^{2}
$$




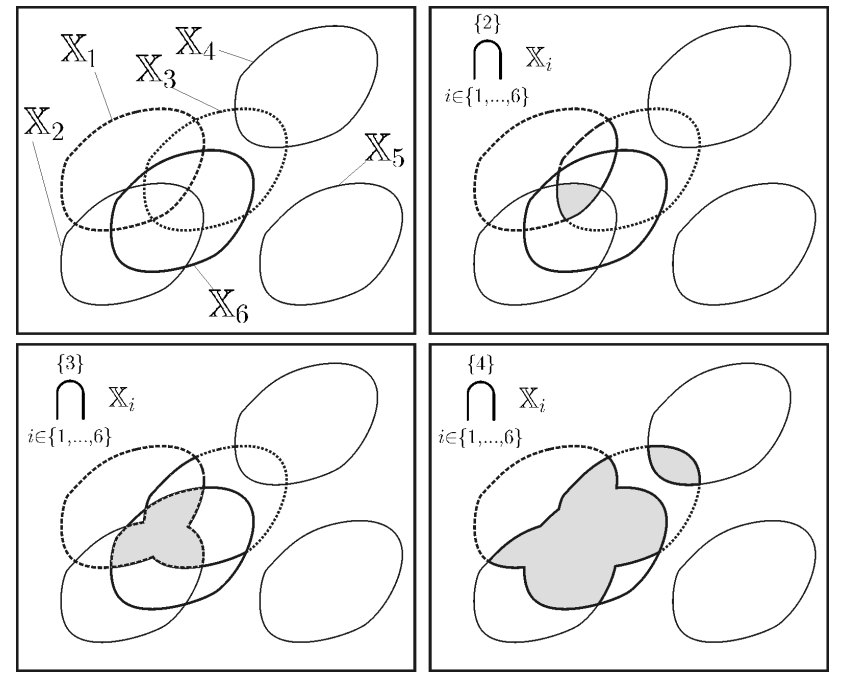

Fig. 1. Illustration (in grey) of the $q$-relaxed intersection of the 6 sets $\mathbb{X}_{1}, \ldots, \mathbb{X}_{6}$ where $q \in\{2,3,4\}$

Define by induction the following notations

$$
\begin{cases}\mathbf{f}_{k: k}(\mathbb{X}) & \stackrel{\text { def }}{=} \mathbb{X} \\ \mathbf{f}_{k_{1}: k_{2}+1}(\mathbb{X}) & \stackrel{\text { def }}{=} \mathbf{f}_{k_{2}}\left(\mathbf{f}_{k_{1}: k_{2}}(\mathbb{X}), \mathbb{N}\left(k_{2}\right)\right), k_{1} \leq k_{2} .\end{cases}
$$

The set $\mathbf{f}_{k_{1}: k_{2}}(\mathbb{X})$ represents the set of all $\mathbf{x}\left(k_{2}\right)$, that are consistent with the fact that $\mathbf{x}\left(k_{1}\right) \in \mathbb{X}$. Consider the following set state estimator

$$
\begin{cases}\mathbb{X}(k)= & \mathbf{f}_{0: k}(\mathbb{X}(0)) \quad \text { if } k<m, \text { (initialization step) } \\ \mathbb{X}(k)= & \mathbf{f}_{k-m: k}(\mathbb{X}(k-m)) \cap \\ & \bigcap_{i \in\{1, \ldots, m\}}^{\{q\}} \mathbf{f}_{k-i: k} \circ \mathbf{g}_{k-i}^{-1}(\mathbb{Y}(k-i)) \text { if } k \geq m\end{cases}
$$

If we assume that (i) within any time window of length $m$ we never have more than $q$ outliers and that (ii) $\mathbb{X}(0)$ contains the true value for $\mathbf{x}(0)$, then $\mathbb{X}(k)$, as defined by (4), corresponds to the set of all feasible $\mathbf{x}(k)$ (see [6]). The principle of the observer (4) is illustrated by Figure 2 for $m=3$ and $q=1$. In this figure, double arrows are used to describe the correspondences between sets. For instance, the rightmost set corresponds to $\mathbf{f}_{k-2: k} \circ \mathbf{g}_{k-2}^{-1}(\mathbb{Y}(k-2))$ and represents the set of all $\mathbf{x}(k)$ that are consistent with the $k-2$ data set. The small grey circles are the true values of the state vectors $\mathbf{x}(k-i)$ and output vectors $\mathbf{y}(k-i)$. Note that $\mathbf{y}(k-2)$ is outside $\mathbb{Y}(k-2)$ and is thus is an outlier, whereas $\mathbf{y}(k-1)$ and $\mathbf{y}(k-3)$ are inliers. The state estimator can efficiently be implemented using an interval constraint propagation approach which recursively computes supersets which enclose the $\mathbb{X}(k)$ 's. 


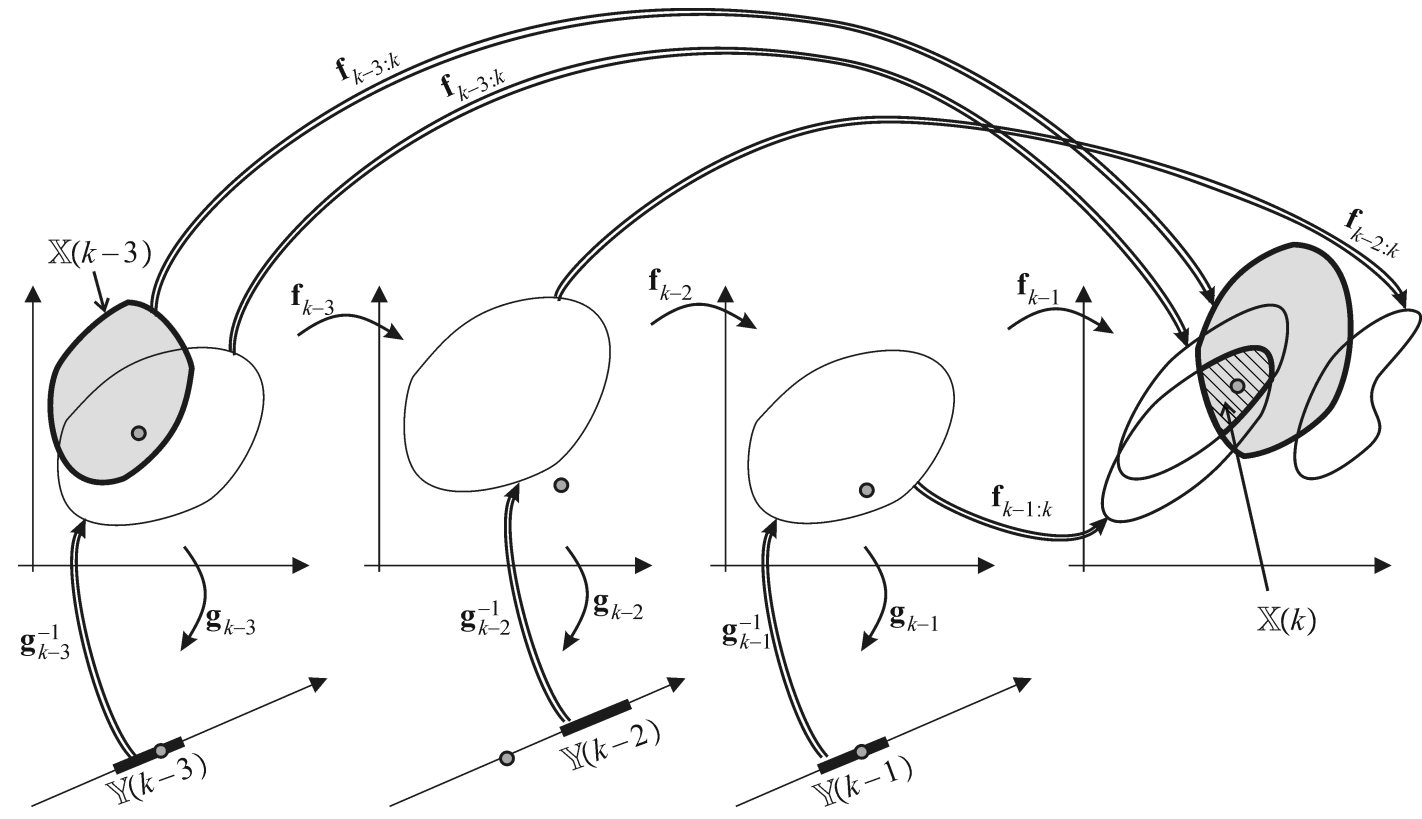

Fig. 2. The feasible set for the state vector $\mathbb{X}(k)$, assuming at most $q=1$ outlier, can be defined recursively from $\mathbb{X}(k-3)$ and from the data sets $\mathbb{Y}(k-1), \mathbb{Y}(k-2), \mathbb{Y}(k-3)$.

\section{PRobabilistic ANALYSis}

This section provides a probabilistic interpretation of the set-membership observer presented in the previous section. We shall assume that all events "y $(k) \in \mathbb{Y}(k) ", k>0$ and the event $\mathbf{x}(0) \in \mathbb{X}(0)$ are all independent, a priori. This assumption can be interpreted as the fact that the occurrence of an outlier at time $k$ is independent from the past, which is close to the classical Markovian assumption. For simplicity, we shall also assume that the known prior probability $\pi_{y}=\operatorname{Pr}(\mathbf{y}(k) \in \mathbb{Y}(k))$ does not depend on $k$.

Proposition. Consider the following hypothesis, denoted by $\mathcal{H}_{q}\left(k_{1}: k_{2}\right)$, which states that among all $k_{2}-k_{1}+1$ output vectors, $\mathbf{y}\left(k_{1}\right), \ldots, \mathbf{y}\left(k_{2}\right)$, at most $q$ of them are outlier. We have

$$
\operatorname{Pr}\left(\mathcal{H}_{q}(k-m: k-1)\right)=\sum_{i=m-q}^{m} \frac{m !}{i !(m-i) !} \pi_{y}^{i} \cdot\left(1-\pi_{y}\right)^{m-i} .
$$

Proof. The prior probability of having exactly $i$ inliers among $m$ follows a binomial distribution given by

$$
\beta\left(i, m, \pi_{y}\right)=\frac{m !}{i !(m-i) !} \pi_{y}^{i} \cdot\left(1-\pi_{y}\right)^{m-i} .
$$

Thus, the probability of having at least $m-q$ inliers (or equivalently having at most $q$ outliers) among $m$ data is $\sum_{i=m-q}^{m} \beta\left(i, m, \pi_{y}\right)$. 
Theorem. Consider the sequence of sets $\mathbb{X}(0), \mathbb{X}(1), \ldots$ built by the observer (4). We have

$$
\begin{aligned}
\operatorname{Pr}(\mathbf{x}(k) \in \mathbb{X}(k)) \geq & \operatorname{Pr}(\mathbf{x}(k-m) \in \mathbb{X}(k-m)) * \\
& \sum_{i=m-q}^{m} \frac{m ! \pi_{y}^{i} \cdot\left(1-\pi_{y}\right)^{m-i}}{i !(m-i) !} .
\end{aligned}
$$

Moreover, in the special case where $\mathbb{N}(k)$ are all singletons (which amounts to saying that we have no state noise) and the functions $\mathbf{f}_{k}$ are all injective, then the inequality becomes an equality.

Proof. Since the $m+1$ following events:

$$
\mathbf{x}(k-m) \in \mathbb{X}(k-m) \text { and }\left\{\begin{aligned}
& \mathbf{y}(k-m) \in \mathbb{Y}(k-m) \\
& \vdots \\
& \mathbf{y}(k-1) \in \mathbb{Y}(k-1)
\end{aligned}\right.
$$

are all independent and since

$$
\begin{aligned}
\mathbb{X}(k) \stackrel{\text { def }}{=} & \mathbf{f}_{k-m: k}(\mathbb{X}(k-m)) \cap \\
& \bigcap_{i \in\{1, \ldots, m\}}^{\{q\}} \mathbf{f}_{k-i: k} \circ \mathbf{g}_{k-i}^{-1}(\mathbb{Y}(k-i)) \quad \text { if } k \geq m
\end{aligned}
$$

we have the following implication

$$
\left.\begin{array}{c}
\mathbf{x}(k-m) \in \mathbb{X}(k-m) \\
\mathcal{H}_{q}(k-m: k-1)
\end{array}\right\} \Rightarrow \mathbf{x}(k) \in \mathbb{X}(k)
$$

Since the two events $\mathbf{x}(k-m) \in \mathbb{X}(k-m)$ and $\mathcal{H}_{q}(k-m: k-1)$, are independent, we have

$$
\operatorname{Pr}(\mathbf{x}(k) \in \mathbb{X}(k)) \geq \operatorname{Pr}(\mathbf{x}(k-m) \in \mathbb{X}(k-m)) * \operatorname{Pr}\left(\mathcal{H}_{q}(k-m: k-1)\right)
$$

and thus

$$
\operatorname{Pr}(\mathbf{x}(k) \in \mathbb{X}(k)) \geq \operatorname{Pr}(\mathbf{x}(k-1) \in \mathbb{X}(k-1)) * \sqrt[m]{\operatorname{Pr}\left(\mathcal{H}_{q}(k-m: k-1)\right)}
$$

Assume that we have no state noise and that the $\mathbf{f}_{k}$ are all injective. The implication (6) becomes an equivalence and thus (7) becomes an equality.

\section{ApPlicAtion to LOCALIZATION}

As an illustration, we shall now consider the problem of the localization and control of an underwater robot. The problem is similar to that presented in [6], but here, we shall add the probabilistic information. 
Set-membership methods have often been considered for robot localization (see, e.g., [13], in the case where the problem is linear and also [3] when the robot is underwater). In situations where strong nonlinearities are involved, interval analysis has been shown to be particularly useful (see, e.g., [12], [4]). Here, the approach is made more efficient by the addition of constraint propagation techniques. Assume the robot evolution is described by

$$
\left\{\begin{array}{l}
\dot{x}_{1}=x_{4} \cos x_{3} \\
\dot{x}_{2}=x_{4} \sin x_{3} \\
\dot{x}_{3}=u_{2}-u_{1} \\
\dot{x}_{4}=u_{1}+u_{2}-x_{4},
\end{array}\right.
$$

where $x_{1}, x_{2}$ are the coordinates of the robot center, $x_{3}$ is its orientation (see Fig. 3) and $x_{4}$ is its speed. The inputs $u_{1}$ and $u_{2}$ are the accelerations provided by the left and right propellers, respectively. This model corresponds to an underwater robot with a constant depth (the depth regulation of the robot is assumed to be already solved and will not be considered here) and with no roll and pitch. Thus, our robot can be seen as a two-dimensional robot. The localization problem for this type of robot in the presence of outliers is similar to that treated in [12] or [8], but, in these two papers, the outliers was treated with a static manner, i.e., at each $k$ a lot of measurements were collected (24 sensors were available for the application treated). The robot pose had to be consistent with all measurements made at time $k$ except $q$ of them. In [6], the outliers was treated in a dynamic way, but no probability was given on the resulting set. The system can be discretized by $\mathbf{x}_{k+1}=\mathbf{f}_{k}\left(\mathbf{x}_{k}\right)$ where,

$$
\mathbf{f}_{k}\left(\begin{array}{c}
x_{1} \\
x_{2} \\
x_{3} \\
x_{4}
\end{array}\right)=\left(\begin{array}{c}
x_{1}+\delta \cdot x_{4} \cdot \cos \left(x_{3}\right) \\
x_{2}+\delta \cdot x_{4} \cdot \sin \left(x_{3}\right) \\
x_{3}+\delta \cdot\left(u_{2}(k)-u_{1}(k)\right) \\
x_{4}+\delta \cdot\left(u_{1}(k)+u_{2}(k)-x_{4}\right)
\end{array}\right)
$$

and $\delta=0.01 \mathrm{sec}$ is the sampling time. The robot moves inside a swimming pool with a known shape (four vertical planar walls and two vertical cylinders). The robot is equipped with a sonar which measures the horizontal distance between the robot and the border of the pool following the direction pointed by the sonar. The sonar turns around itself (see Fig. 3) with an angular speed of $5 \mathrm{rad} / \mathrm{sec}$. Denote by $\alpha(k)$ the angle between the direction of the sonar and the axis of the robot. Since the swimming pool is composed with vertical walls, the observation equation of the system has the form $d=g_{k}(\mathbf{x})$. Even if the functions $\mathbf{f}_{k}$ 


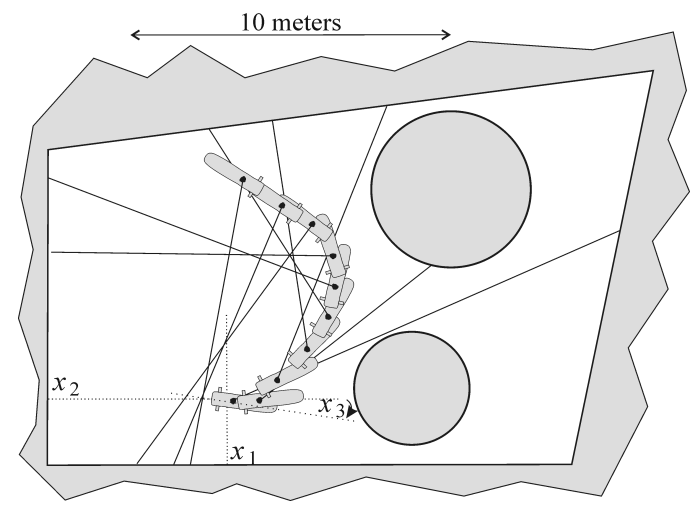

Fig. 3. Underwater robot moving inside a pool

and $g_{k}$ are strongly nonlinear, the feasible set $\mathbb{X}_{k}$ can efficiently be characterized using interval propagation methods. The Tchebychev center $\hat{\mathbf{x}}_{k}$ of $\mathbb{X}_{k}$ (i.e., the center of the smallest cube enclosing $\mathbb{X}_{k}$ ) is returned by our observer as an estimation of the actual state vector for the robot. This estimate is then used by the controller to compute the values $\mathbf{u}$ to be given to the propellers.

Consider now a mission for the robot where three waypoints have to be reached. Once a waypoint is thought to be reached with a precision less than $0.5 \mathrm{~m}$, the planner sends the next waypoint, until all waypoints have been reached. The length of the sliding time window is chosen as $m=100$, which corresponds almost to one complete turn of the sonar. The number of allowed outliers inside a time window of length $m$ is chosen as $q=60$. In our simulation, an outlier is generated with a probability of 0.5 . In order to facilitate the visualization of the results, when an outlier is generated, the measured distance returned by the simulated robot is fixed at the unknown distance of $15 \mathrm{~m}$. Moreover, to the measured distance, we added a white noise with a uniform distribution inside the interval $[-0.03,0.03]$, which corresponds to an error of $\pm 3 \mathrm{~cm}$. Figure 4 illustrates the mission of the robot for $t \in\{3,6,9,12,15,16.2\}$ where $16.2 \mathrm{sec}$ corresponds to the duration of the mission. The black squares represent the current waypoint where the robot plans to go. The grey segments correspond the sonar distances estimated by our observer. Note that here these segments also correspond to the true distances. The small black circle represent the current position of the robot. The associated black tail represents all positions the robot had in the time interval $[t-m \delta, t]$.A typical emission diagram, associated to $t=9 \mathrm{sec}$, is represented on Figure 5 . The 42 outliers correspond to the grey segments. The black segments correspond to filtered distances that have been returned by our observer. 


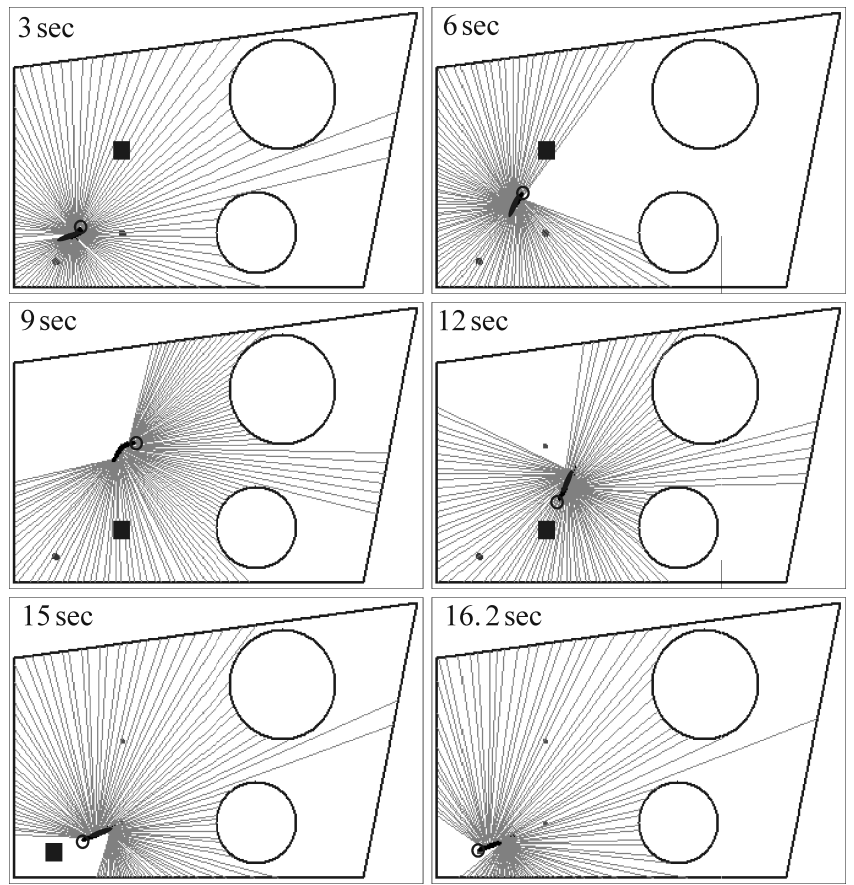

Fig. 4. Illustration of the robot mission for different times $t$

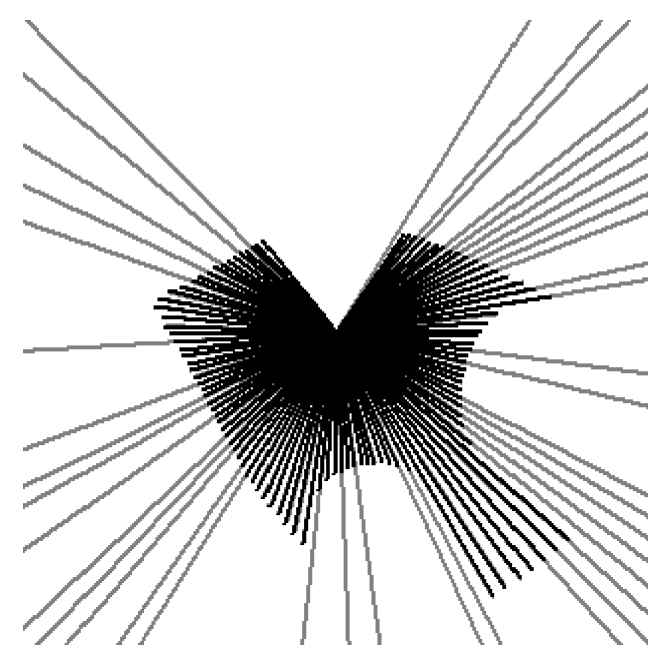

Fig. 5. Emission diagram at time $t=9 \mathrm{sec}$

The actual trajectory as well as the set-membership envelope returned by the observer are depicted on Figure 6.

For different times $t$, the table below provides a lower bound for $\operatorname{Pr}(\mathbf{x}(k) \in \mathbb{X}(k))$ and the (unknown) number of outliers that are stored inside the current data buffer of the observer. 


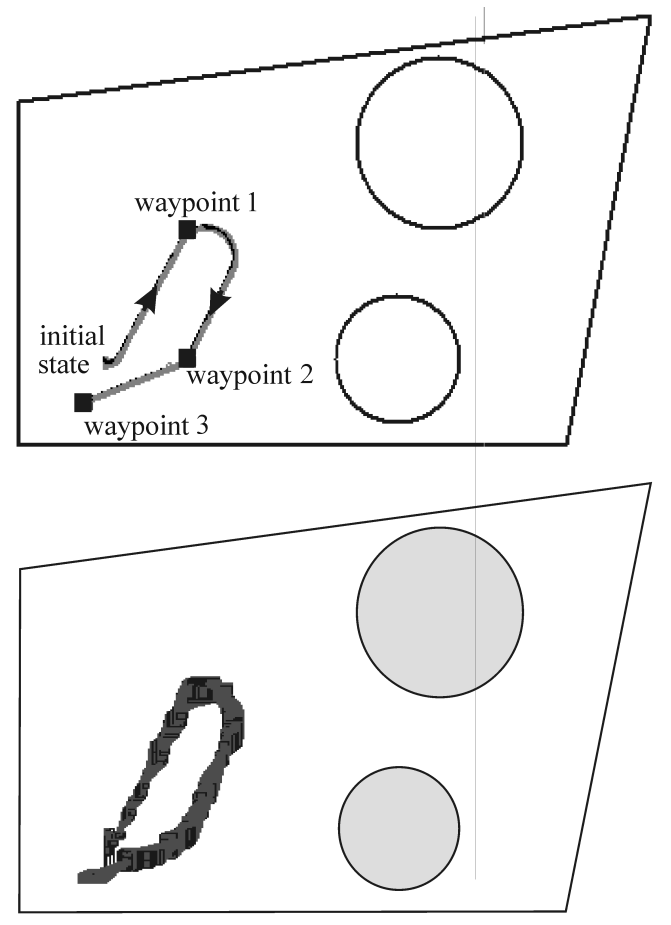

Fig. 6. Actual trajectory of the robot and the corresponding envelope

\begin{tabular}{|c|c|c|}
\hline$t(\mathrm{sec})$ & $\operatorname{Pr}(\mathbf{x} \in \mathbb{X})$ & Outliers \\
\hline 3.0 & $\geq 0.965$ & 58 \\
6.0 & $\geq 0.932$ & 50 \\
9.0 & $\geq 0.899$ & 42 \\
12.0 & $\geq 0.869$ & 51 \\
15.0 & $\geq 0.838$ & 51 \\
16.2 & $\geq 0.827$ & 49 \\
\hline
\end{tabular}

The computation time for all the mission takes less than $100 \mathrm{sec}$ on classical personal computer, which makes the approach consistent with real time applications. The $\mathrm{C}++$ Builder 5 source codes of this test case are available at the following address

www.ensieta.fr/jaulin/probintk.html 


\section{CONCLUSION}

In this paper, we have proposed a new approach for state estimation which combines an interval setmembership approach with probabilities. This approach has several advantages over classical approaches. By propagating the assumptions on the possible outliers through time, the observer has been made robust with respect to a large number of outliers. Moreover, thanks to interval analysis, the observer is able to deal with nonlinear (or non-differentiable and even noncontinuous) state equations, without linearizing or approximating them. But the remarkable property of our observer is its ability to provide a probability associated with the current set $\mathbb{X}(k)$ for the state vector $\mathbf{x}(k)$. This is new in the context of set-membership estimation. As a consequence, the observer was able to take into account the fact that there always exists a nonzero probability that some of the set-membership assumptions are not fulfilled. The principle of the approach has been illustrated on the localization of an underwater robot where many outliers occurred during the mission.

\section{REFERENCES}

[1] F. Abdallah, A. Gning, And P. Bonnifait. Box particle filtering for nonlinear state estimation using interval analysis. Automatica 44(3), 807-815 (2008).

[2] D. P. Bertsekas And I. B. Rhodes. Recursive state estimation for a set-membership description of uncertainty. "IEEE Transactions on Automatic Control" 16(2), 117-128 (1971).

[3] A. Caiti, A. Garulli, F. Livide, And D. Prattichizzo. Set-membership acoustic tracking of autonomous underwater vehicles. Acta Acustica united with Acustica 5(88), 648-652 (2002).

[4] A. Clérentin, M. Delafosse, L. Delahoche, B. Marhic, and A. Jolly-Desodt. Uncertainty and imprecision modeling for the mobile robot localization problem. Autonomous Robots 24(3), 1573-7527 (2008).

[5] A. GNing AND P. Bonnifait. Constraints propagation techniques on intervals for a guaranteed localization using redundant data. Automatica 42(7), 1167-1175 (2006).

[6] L. JAUlin. Robust set membership state estimation ; application to underwater robotics. Automatica 45(1), 202-206 (2009).

[7] L. Jaulin, M. Kieffer, I. Braems, And E. Walter. Guaranteed nonlinear estimation using constraint propagation on sets. International Journal of Control 74(18), 1772-1782 (2001).

[8] M. Kieffer, L. Jaulin, E. Walter, And D. Meizel. Robust autonomous robot localization using interval analysis. Reliable Computing 6(3), 337-362 (2000).

[9] V. Kreinovich, L. Longpré, P. Patangay, S. Ferson, and L. Ginzburg. Outlier detection under interval uncertainty: Algorithmic solvability and computational complexity. In I. LiRKov, S. MARgENOV, J. WASNiEWSKI, AND P. YALAMOV, editors, “Large-Scale Scientific Computing”, Proceedings of the 4th International Conference LSSC'2003 (2003).

[10] S. Lagrange, L. Jaulin, V. Vigneron, and C. Jutten. Nonlinear blind parameter estimation. IEEE TAC 53(4), 834-838 (2008). 
[11] H. LAhanier, E. WALTER, AND R. GOMENi. OMNE: a new robust membership-set estimator for the parameters of nonlinear models. Journal of Pharmacokinetics and Biopharmaceutics 15, 203-219 (1987).

[12] D. Meizel, O. LÉvÊQue, L. JAulin, AND E. WAlter. Initial localization by set inversion. IEEE transactions on robotics and Automation 18(6), 966-971 (2002).

[13] D. Meizel, A. Preciado-Ruiz, and E. Halbwachs. Estimation of mobile robot localization: geometric approaches. In M. Milanese, J. Norton, H. Piet-Lahanier, And E. Walter, editors, "Bounding Approaches to System Identification", pp. 463-489. Plenum Press, New York, NY (1996).

[14] R. E. Moore. "Methods and Applications of Interval Analysis". SIAM, Philadelphia, PA (1979).

[15] J. Norton And S. Verez. Outliers in bound-based state estimation and identification. Circuits and Systems 1, 790-793 (1993).

[16] L. Pronzato and E. Walter. Robustness to outliers of bounded-error estimators and consequences on experiment design. In M. Milanese, J. Norton, H. Piet-Lahanier, And E. Walter, editors, "Bounding Approaches to System Identification", pp. 199-212, New York (1996). Plenum.

[17] T. Raissi, N. RAmDAni, AND Y. CANDAU. Set membership state and parameter estimation for systems described by nonlinear differential equations. Automatica 40, 1771-1777 (2004).

[18] M. van EMden. Algorithmic power from declarative use of redundant constraints. Constraints 4(4), 363-381 (1999). 